\title{
Pluralidad política y diversidad cultural. Discursos sobre la educación para la ciudadanía en la sociedad vasca
}

\author{
Txoli Mateos \\ Julen Zabalo \\ Ane Larrinaga \\ Mila Amurrio
}

Universidad del País Vasco. Euskal Herriko Unibertsitatea

Departamento de Sociología y Trabajo Social. Miembros del Grupo de Investigación Parte

Hartuz (IT-697-13)

txoli.mateos@ehu.eus; julen.zabalo@ehu.eus; ane.larrinaga@ehu.eus; mila.amurrio@ehu.eus

Recibido: 08-04-2014

Aceptado: 25-03-2015

\section{Resumen}

La sociedad vasca es políticamente plural —y escenario de conflicto sobre su estatus político- - además de culturalmente diversa, e inmersa, asimismo, en un permanente debate sobre el papel que se debe asignar al idioma y a la cultura vascos en relación con la cultura desarrollada en español. En este artículo, indagamos en las visiones sobre la asignatura denominada educación para la ciudadanía y los derechos humanos, desarrolladas por las principales organizaciones sindicales y políticas vascas, así como por algunos profesores de enseñanza no universitaria. Mediante entrevistas en profundidad a los primeros y grupos de discusión con los segundos, analizamos el discurso generado en torno a la educación cívica como medio indirecto de hallar la respuesta a tres temas cruciales para la vida social vasca: el concepto de ciudadanía, la visión sobre la nación y la convivencia entre culturas. Y destacamos una serie de conclusiones: $a$ ) la falta de significatividad política en relación con la identidad nacional que la asignatura recibe, exceptuando a algunas organizaciones nacionalistas vascas; $b$ ) la preeminencia del discurso moral de corte liberal en todos los agentes políticos y educativos; $c$ ) la aceptación generalizada de la diversidad cultural como elemento enriquecedor, aunque con diferencias notorias entre profesores y organizaciones respecto a sus consecuencias educativas, y d) la desconfianza del nacionalismo vasco y de un sector del profesorado hacia un discurso multiculturalista que signifique el retroceso de la lengua vasca.

Palabras clave: educación cívica; liberalismo; nacionalismo vasco; nacionalismo español; multiculturalismo; País Vasco 
Abstract. Political plurality and cultural diversity. Discourses on citizenship education in basque society

Basque society is politically plural — as well as being a scene of conflict over its political status - , culturally diverse, and caught up in a permanent debate on the role to be assigned to the Basque language and culture in relation to the development of culture in Spanish. This article will examine the different views on the subject Education for Citizenship and Human Rights developed by the main Basque trade union and political organizations, together with a group of non-university teachers. With the aid of in-depth interviews with the former, and focus groups with the latter, we shall analyse the discourse generated on civic education as an indirect means to finding answers to three crucial subjects for Basque society: the concept of citizenship, the view of the nation and co-existing cultures. In addition, we shall point out some conclusions: $a$ ) in relation to national identity, no political significance is afforded to the subject, except in the case of certain Basque nationalist organisations; b) a liberal moral discourse is predominant among all the political and educational players; $c$ ) cultural diversity is generally accepted as something enriching, although there is significant divergence between teachers and the different organisations as to its educational consequences, and $d$ ) there is mistrust on the part of the Basque nationalist movement and some teachers of the multiculturalist discourse as they see it as a setback for the Basque language.

Keywords: civic education; liberalism; Basque nationalism; Spanish nationalism; multiculturalism; Basque Country

\author{
Sumario \\ 1. Introducción \\ 2. Los dilemas de la educación cívica: \\ ciudadanía, nacionalidad \\ y diversidad cultural \\ 3. Descripción metodológica
}

\title{
1. Introducción
}

El comienzo de la redacción de este artículo coincidió con la aprobación, por parte del Consejo de Ministros del Gobierno español, de la tramitación de una nueva ley educativa (LOMCE), entre cuyas novedades se encontraba, precisamente, la desaparición de la asignatura llamada educación para la ciudadanía $y$ los derechos humanos (ECDH), objeto de estudio de la investigación que se encuentra en la base del presente texto. En un primer momento, el ministro de Educación — quien anteriormente había sido el centro de una gran polémica al afirmar que su objetivo era españolizar a las generaciones jóvenes- propuso, sin éxito, su sustitución por una asignatura llamada educación cívica y constitucional, donde se hacía mención expresa al nacionalismo excluyente, en clara referencia a los nacionalismos vasco y catalán. Valores sociales y cívicos, en la educación primaria, y valores éticos, en la secundaria obligatoria, que harán las 
veces de alternativa a la asignatura de religión, se pueden considerar las asignaturas más cercanas a la ECDH. La desaparición de esta refleja claramente hasta qué punto ha sido, desde su implantación en 2006, una asignatura controvertida y polémica (Soriano, 2008, y, sobre todo, Lorenzo Alquézar, 2010), así como, en el caso de los sectores conservadores, un tema realmente incómodo, especialmente en su dimensión moral y religiosa (entre otros, Ceballos et al., 2009). Con la aprobación de dicha ley, España se sitúa fuera de una tendencia cada vez más extendida y ya ha empezado a cosechar críticas, incluso desde los futuros profesionales de la enseñanza (Molina Puche et al., 2013: 114-116).

Hace ya tiempo que se da por sentada la necesidad de que los jóvenes sean formados como ciudadanos, y se adjudica a la Administración pública un alto grado de responsabilidad en esta función. De hecho, la formación de ciudadanos - nacionales - ha sido uno de los pilares de la construcción del Estado moderno, así como el motor para la extensión e institucionalización de los sistemas educativos tal como los entendemos hoy en día, en su versión más clásica (Green, 1990; Ramírez y Boli, 1999; Wiborg, 2000) o en creaciones más recientes (Pedazhur, 2001). La educación para la ciudadanía lleva más de una década integrada en los currículos de los sistemas educativos en el ámbito occidental y fuera de este. Además, es frecuentemente tema de controversia y reflexión, tanto en el terreno educativo como en el académico y político, aunque la asignatura no tiene el mismo estatus, responde a diferentes contextos culturales, no refleja las mismas preocupaciones morales y políticas, no ocupa idéntico lugar en el currículo, ni, lógicamente, muestra los mismos contenidos y objetivos (Kerr, 2012; Schulz et al., 2010; Schwille y Amadeo, 2002; Torney-Purta, 2002).

La caída del Muro de Berlín y la consiguiente puesta en marcha de programas para aprender a vivir democráticamente, además de la crítica comunitarista al liberalismo (Naval, 2003: 170-171); el declive del capital social (Kisby, 2006); la crisis de las instituciones democráticas tradicionales junto a una euforia participativa presente en el auge del modelo deliberativo como en el discurso de las instituciones públicas (García Guitián, 2008: 80-81), son algunos de los orígenes adjudicados a la relevancia de este tema, reflejada en la producción de científicos sociales de diversos ámbitos que llevan años resaltando la importancia de la educación para la ciudadanía (Bárcena, 1999; Callan, 2004; Gutmann, 2001; Kymlicka, 2003; Naval, 1995; Pearce y Hallgarten, 2000).

Es evidente que la atracción que ejerce el devenir de esta materia en el sistema educativo de las democracias liberales, e incluso su consideración como elemento crucial en las transiciones democráticas (Faour y Muasher, 2011; Waddell, 2013) o su incierto papel en las democracias no liberales (Pedazhur, 2001) tiene que ver con la importancia que ha adquirido el término ciudadanía y con el interés en su transmisión a las generaciones más jóvenes. Sin embargo, mientras que, en algunos países —el Reino Unido, por ejemplo-, la introducción en el currículo de la educación para la ciudadanía como asignatura obligatoria se consideró un hito en la historia de la educación (Andrews y Mycok, 2007: 73), o la iniciativa educativa más importante tomada por el 
gobierno en muchos años (Kisby, 2006: 151), en el caso de la sociedad vasca, puede decirse que ha pasado casi desapercibida ${ }^{1}$.

\section{Los dilemas de la educación cívica² ${ }^{2}$ ciudadanía, nacionalidad y diversidad cultural}

Parece existir un consenso bastante generalizado acerca de considerar que es una tarea urgente educar ciudadanos que compartan una identidad suficientemente cohesiva en el marco de sociedades cada vez más plurales. La creación de nuevas formas de comunidad es uno de los retos más citados. Ello refleja la preocupación por la falta de interés e implicación de las generaciones jóvenes en la vida pública y política, lo que se considera un serio déficit democrático (Kerr, 1999: 12). Tanto es así que se habla de la educación cívica como un derecho fundamental del alumnado, puesto que la asignatura tiene el valor intrínseco de hacerle entender temas que le afectan directamente y de dotarle de capacidades para participar en la resolución democrática de las decisiones (Andrew y Mycock, 2007: 73).

Sin embargo, cuando los contenidos relacionados con la educación de la ciudadanía aparecen explicitados en una asignatura, esta tiene a menudo menor estatus que el resto de asignaturas, y se le ofrece menos tiempo que a las demás (Schwille y Amadeo, 2002: 111). A pesar de las continuas llamadas a poner en práctica o a realizar mejoras en esta materia, la verdad es que no resulta una tarea fácil, entre otras cosas, porque la función política que supuestamente debe cumplir la educación de la ciudadanía aparece como secundaria frente a las demandas dirigidas a la escuela de cara al mercado laboral y al mundo profesional (Pedró, 2003: 238). Sería necesario un profundo cambio en la concepción del sistema educativo para que surtiesen realmente efecto los discursos que ponen de manifiesto la importancia de la educación cívica.

Pero, unido a esto, en las sociedades democráticas liberales, la educación para la ciudadanía se enfrenta a diversas tensiones o dilemas — según las diversas denominaciones - que estaría obligada a resolver. En el contexto europeo, una serie de áreas potenciales de tensión en torno a la educación cívica hacen referencia al posible conflicto entre el papel de ciudadano y el de consumidor, la articulación de las identidades políticas nacionales frente a una identidad europea, o la identidad política y cívica frente a otras identidades religiosas o culturales (Pedró, 2003: 239). En el contexto más amplio de las sociedades occidentales, el modelo de ciudadanía liberal se halla enfrentado a dos grandes tipos de desafíos, que afectan directamente a la concepción de la educación cívica. Uno hace referencia al contenido liberal de la ciudadanía, en la medida

1. Genera polémica, sin embargo, la oferta del Gobierno de la Comunidad Autónoma Vasca (PSE-EE y después PNV) al alumnado de cuarto curso de la ESO y de bachiller de unos módulos llamados educación para la paz, que pretenden dar testimonio de las víctimas de la violencia (hasta el momento, de ETA y GAL; a partir del curso 2013-2014, también víctimas de abusos policiales).

2. En el texto, utilizamos educación para la ciudadanía y educación civica como sinónimos. 
en que, por ejemplo, el fundamentalismo religioso considera normas liberales como el laicismo o la tolerancia una amenaza para su forma de vida. Son un tipo de controversias sobre qué verdades deberían ser enseñadas y que, normalmente, están basadas en la identidad o en la falta de reconocimiento de algún grupo social, por ejemplo: cristianos o gays y lesbianas (Appiah, 2003). El segundo reto hace referencia a la escala nacional de la ciudadanía y lo plantean los grupos nacionales subestatales que se muestran en desacuerdo con otorgar al estado nación correspondiente el carácter de comunidad política prioritaria (Kymlicka, 2002). En este caso, la ciudadanía, la identidad cultural, las estructuras gubernamentales y la propia política constituyen temas controvertidos (Smith, 2003).

En lo que respecta al interés de nuestra investigación, hemos agrupado los dilemas a los que se enfrenta el discurso sobre la educación para la ciudadanía en la sociedad vasca en torno a tres bloques que hacen referencia, sucesivamente, a los objetivos morales de la educación cívica, a la escala territorial (nacional, estatal, subestatal, europea, cosmopolita, etc.) en la que se plantea y, por último, a los modelos propuestos de gestión de la diversidad cultural de la sociedad vasca actual.

\subsection{Primer dilema: ¿Qué ciudadanos?}

Como afirma Amitai Etzioni, desde el punto de vista sociológico, está fuera de lugar el debate acerca de si las escuelas deberían comprometerse con la educación moral. No existe educación libre de valores, y las escuelas solo se diferencian entre ellas según el grado de consciencia y franqueza sobre los valores que transmiten (Etzioni, 1999: 219), pero la discusión académica sobre lo que significa ser un (buen) ciudadano parece interminable, porque, lógicamente, está en estrecha relación con las visiones, a veces contrapuestas, sobre la ciudadanía y sobre el grado de compromiso moral que es necesario promover entre los ciudadanos y, por lo tanto, en el sistema educativo. Pablo da Silveira nos habla de tres paradigmas alternativos: el paradigma terapéutico, que adjudica a la educación para la ciudadanía un objetivo de regeneración moral; el paradigma de las reglas, donde el alumnado debe asimilar competencias para poder funcionar en el diseño institucional de la sociedad, $y$, por último, el paradigma de las virtudes cívicas, donde, junto a los objetivos del segundo, se trata de que las nuevas generaciones no se conviertan sólo en ciudadanos, sino también en buenos ciudadanos (Da Silveira, 2009).

Algunos académicos lo afirman rotundamente: la misión de la escuela pública es crear ciudadanos que compartan valores comunes (Bolívar, 2004: 23). En lo que respecta a la educación para la ciudadanía, es precisamente la dimensión referente a los valores cívicos y/o morales que debería transmitir — frente a su papel en la configuración de identidades políticas y culturales- la que más producción acumula a nivel académico. Hablando en términos generales, los estudios sobre los comportamientos y los valores cívicos exceden en mucho los trabajos empíricos que analizan el papel de la escuela en la configuración de 
identidades políticas y culturales (Pedró, 2003: 244). Un somero examen de los decretos oficiales que regulan las competencias y los contenidos, así como, en suma, el papel de la educación para la ciudadanía en el currículo ${ }^{3}$, tanto a nivel español como vasco, pone de manifiesto la preeminencia de la dimensión moral o cívica de la ECDH frente a la cultural o política. Como tendremos ocasión de ver más adelante, en nuestra investigación, es esta dimensión moral y cívica la que más consenso genera en los discursos de nuestros informantes.

Sin duda, es evidente el acento puesto en las sociedades democráticas en la necesidad de fortalecer la participación (e identificación) de sus ciudadanos en el sistema democrático mediante la promoción de la virtud cívica, desde la necesidad de cultivar el carácter (Lickona, 2000), hasta la propuesta de creación de una nueva cultura cívica que transforme al ciudadano mediante el ejercicio habitual de virtudes públicas como la tolerancia, la solidaridad o la responsabilidad (Cortina, 1997). La democracia se convierte, así, no solo en una forma de gobierno, sino también en una forma de vida y va más allá de una educación cívica formal (Barber, 2004; Bárcena, 1999; Peña, 2009). Pero no sólo la democracia, sino incluso la puesta en práctica de los valores estrictamente liberales se considera inviable si no se es capaz de canalizar eficazmente la educación cívica (Galston, 1993: 102).

Según Will Kymlicka (2002: 120-122), el nacionalismo minoritario que ha podido ejercer el autogobierno, lo ha hecho, generalmente, conforme a los valores liberales. Es decir, lo que este tipo de nacionalismo pone en duda es la escala de aplicación de la educación cívica, no su contenido liberal ¿Cuáles son las fronteras de la comunidad política dentro de las que los ciudadanos deben ejercitar las virtudes y los derechos liberales?

\subsection{Segundo dilema: Ciudadanos ;de dónde?}

A primera vista, podría decirse que el estado nación se ha vaciado de contenido en un doble sentido. Por una parte, la globalización económica le ha privado de su capacidad para gestionar algunos asuntos - por ejemplo, los relativos al medio ambiente- que reclaman una gestión que trasciende sus fronteras territoriales. Por otra, se multiplican los procesos de traspaso de competencias o, incluso, de conflictos en torno al reconocimiento de identidades nacionales subestatales - como es el caso de Cataluña y el País Vasco, con España-. Ambos procesos repercuten directamente en la soberanía y la naturaleza de la identidad nacional. Es lo que David Miller ha llamado fragmentación de la identidad política o de ese sentido de pertenencia fuerte que acompañó al proceso de creación del estado nación. Ya no sabemos si somos ciudadanos de un solo estado o de una serie de comunidades políticas interconectadas (Miller, 2000).

Según Anthony Giddens, estamos asistiendo al surgimiento de una nación cosmopolita, cuya existencia debe tener un efecto directo sobre la educación de

3. El Real Decreto 1513/2006 fija los contenidos mínimos comunes a todo el territorio español de la materia de educación para la ciudadanía y los derechos humanos. 
sus ciudadanos, porque uno de sus componentes fundamentales debe ser la tolerancia de la identidad múltiple. Una nación cosmopolita es aquella que capacita a todos sus ciudadanos para vivir cómodamente con identidades diversas, es decir, para ser inglés, británico, europeo y quizá ciudadano de otra sociedad mundial emergente (Giddens, 2000). Los defensores más radicales de la dimensión cosmopolita de la educación cívica piensan que el objetivo de esta es la promoción de una ciudadanía universal, superando así el marco del estado nación, lo que conllevaría el abandono de los localismos y el reforzamiento de los lazos entre todos los seres humanos (Cortina, 1997; Rosales, 2000; Nussbaum, 1999). En este sentido, parece que la formación de la identidad nacional, que fue una vez el requisito esencial de la educación cívica en muchos países, no posee excesivo protagonismo en el siglo XXI, aunque se puedan citar excepciones remarcables, como es el caso de los Estados Unidos de América (Schwille y Amadeo, 2002: 130).

No obstante, algunos académicos consideran que, pese a la globalización económica y cultural, el estado nación sigue manteniendo una función relevante. Una simple mirada a las políticas educativas de las naciones europeas, por ejemplo, muestra que estas todavía tienen objetivos nacionales (Wiborg, 2000). En el plano normativo, tanto en el pensamiento republicano (Colom Cañellas y Rincón Verdera, 2007; Schnapper, 2001) como el más próximo al comunitarismo (Etzioni, 1999; MacIntyre, 1993), estiman que la escuela es la institución de la nación por excelencia. No muestran ninguna duda respecto a la necesidad de transmitir una historia compartida, algo así como un currículo nacional, un cuerpo central de materiales que todos los niños deberían asimilar.

Así mismo, pensadores liberales de estilos muy diferentes reconocen que, en la búsqueda de compromisos cívicos, es necesario transmitir a las generaciones jóvenes ciertas dosis de patriotismo o, si se prefiere, de orgullo nacional, a través de la narrativa histórica de un panteón de héroes a los que emular. Expresado en otros términos, las escuelas deben verse como lugares en los que se reproduce una identidad nacional común, aunque esta no tenga que estar necesariamente basada en una estricta homogeneidad cultural (Brighouse, 2003; Galston, 1993; Kymlicka, 2003; Miller, 1997). Eso significa que, en el marco de la educación cívica, el cultivo del sentimiento nacional puede ser respetuoso con los ideales de libertad y autonomía individual, lejos del carácter no liberal que se adjudica a veces al nacionalismo (Smith, 2003; Williams, 1995).

Pero al primer dilema entre escoger la transmisión de la identidad nacional o dar preferencia a una identidad cosmopolita se suma un segundo dilema, que supone elegir entre la identidad nacional emanada del estado o, como dice Will Kymlicka, plantear un reto al estado nación, al modo en que lo hacen las minorías nacionales. Estas son escépticas ante cualquier forma de educación cívica que tome a las instituciones estatales como algo dado y como el foro principal de lealtad política. La educación cívica, así, se considera a menudo, sencillamente, como un instrumento de asimilación cultural y política (Kymlicka, 2002: 120). 
De hecho, en algunas sociedades donde no existe consenso en torno a una identidad nacional común, no parece que la educación para la ciudadanía, ni la escuela en sí, sean instrumentos eficaces de resolución del conflicto. Así, en el sistema educativo de Israel, una de las identidades hegemoniza a los contenidos y margina a las otras identidades, transmitiendo una imagen estereotipada de ellas (Ichilov, 2003; Pedazhur, 2001); en Irlanda del Norte, las identidades nacionales se soslayan, mostrándose ambiguas, difusas e indefinidas (Niens et al., 2013: 5), de este modo, impiden la construcción de una visión compartida del conflicto y de sus posibles soluciones (Smith, 2003), y en Bosnia, las diversas identidades nacionales en conflicto impregnan el currículo con su propia visión político-cultural, con lo cual se genera una organización sectaria del sistema educativo (Swimelar, 2012).

Sin embargo, no todos los estudiosos del tema afrontan las relaciones entre nacionalismo y educación en el marco de la educación cívica como un asunto que requiere, fundamentalmente, unión o cohesión. En opinión de Yael Tamir, la solución está en una aplicación más amplia de la democracia liberal, es decir, en permitir que cada comunidad desarrolle los contenidos que considere relevantes. Por tanto, la clave no está en unir, sino en separar la educación cívica de la educación nacional (Tamir, 1993: 68).

\subsection{Tercer dilema: Ciudadanos pertenecientes ¿a qué cultura?}

Tanto la dimensión moral como la dimensión nacional de la educación para la ciudadanía se relacionan directamente con su dimensión cultural, porque es mucho más fácil promover la participación política y llevar a cabo medidas que impliquen un cierto sacrificio y compromiso moral cuando hay una identidad cultural compartida, y los ciudadanos nos sentimos unidos de alguna manera a nuestros conciudadanos. Pero uno de los rasgos más evidentes de las sociedades modernas es su pluralidad cultural y las tensiones que genera la adecuada gestión de esta.

Según algunos teóricos, la historia, la literatura y la lengua de una nación deben ocupar un lugar preferente en el currículo de la escuela pública, porque las políticas democráticas se construyen en base a la identificación cultural de los ciudadanos y estos se implican más fácilmente en la búsqueda de la justicia. No es necesario aducir ninguna superioridad moral, afirma Amy Gutmann. De la misma forma que amamos a nuestros hijos más que a otros niños, valoramos las orientaciones culturales de nuestro país simplemente porque son nuestras y otorgan significado a nuestra vida (Gutmann, 2001: 64). Pero el hecho es que, en muchos países de Europa y fuera de esta, los estudiantes de minorías étnicas experimentan discriminación por sus diferentes características y valores culturales y religiosos; son frecuentemente percibidos como el Otro, lo que genera una débil lealtad al estado nación por parte de ellos (Banks, 2008: 132). Si uno de los objetivos de la educación promovida por el estado nación es que los ciudadanos interioricen la cultura nacional, se puede vislumbrar hasta qué punto se convierte en complejo el dilema entre primar esta o dar 
cabida a una visión multiculturalista de las relaciones entre culturas que suponen los movimientos migratorios actuales.

Entendemos el multiculturalismo como el conjunto de políticas institucionales que, a partir de la consideración como un valor del pluralismo cultural, atienden a las demandas de reconocimiento colectivo de grupos identitarios diversos (Máiz, 2003: 447). En cualquier parte del planeta, los estados intentan determinar si van a continuar con una ideología de asimilación cultural de la inmigración o, por el contrario, se van a considerar a sí mismos sociedades multiculturales y van a permitir a los inmigrantes apropiarse de una ciudadanía multicultural (Kymlicka, 1996). Y aún en el caso de que esta visión de respeto a la identidad cultural de los diferentes grupos sociales pueda ser sólida, la verdad es que la diferencia entre la teoría y la práctica suele ser grande, y las tensiones son innegables, incluso en sociedades con una larga tradición liberal y/o democrática (Doppen, 2010; Innerarity, 2007). En algunas de ellas, se es cada vez más consciente de la necesidad de realizar un replanteamiento de la política multiculturalista en aras de hacer compatible la diferencia cultural con los requerimientos de la democracia, con la igualdad social y los valores compartidos (Gutmann, 2008), abogando porque la educación para la ciudadanía sea educación intercultural (Bolívar, 2004), e, incluso, se piensa que es necesario que las políticas multiculturalistas vayan acompañadas de una identidad nacional fuerte (Asari et al., 2008).

Si esto es así en el caso del estado nación, los dilemas son, si cabe, más complejos al enmarcar las relaciones entre la cultura nacional y la diversidad cultural en el contexto de las sociedades con nacionalismos subestatales, como es el caso de la sociedad vasca (Mateos, 2009; Zabalo et al., 20013). Como señala Ramón Máiz (2003: 434-43), los nacionalismos subestatales pueden tener la tentación de reproducir, siempre a menor escala, la lógica uniformadora del estado nación. Es decir, siendo su objetivo la salvaguarda de su lengua y cultura nacionales, a menudo se ven expuestos a problemas similares a los que originaron sus propias reivindicaciones: ¿Qué hacer con el pluralismo interno de la sociedad? ¿Hasta qué punto hay que respetar los contextos culturales y lingüísticos de las minorías?

\section{Descripción metodológica}

El propósito del presente trabajo ha sido la identificación de los discursos sobre la educación para la ciudadanía que coexisten en la sociedad vasca, que está caracterizada por el pluralismo cultural y la confrontación política, especialmente en lo referente a la identidad nacional. De este modo, se ha optado por la aplicación de técnicas basadas en metodología cualitativa para la obtención de datos, habiéndose realizado tanto entrevistas en profundidad como grupos de discusión, en primer lugar, con la finalidad de estudiar los significados intersubjetivos producidos por agentes políticos y educativos en torno a la educación cívica y, en segundo lugar, con la intención de reconocer y definir los dilemas a los que se enfrenta esta en la sociedad vasca actual. 
Siguiendo la perspectiva cualitativa, se ha realizado una selección estratégica para el diseño de la muestra de la investigación. Cabe recordar que este tipo de muestreo no tiene como objetivo la generalización estadística, sino que, para el diseño de la investigación, posee mayor relevancia la elección de sujetos detentadores de determinadas características que la representatividad de los elementos de la población. Así, los criterios utilizados para la selección de las unidades que completan la muestra han tenido como horizonte la búsqueda de la mayor diversidad posible de visiones, a partir de la consideración de un doble eje: la diferenciación de posiciones político-ideológicas (izquierda-derecha) y la diferenciación de identidades nacionales (española-vasca). Puesto que ambas líneas de división afectan tanto a la sociedad de la Comunidad Autónoma Vasca como a la de la Comunidad Foral de Navarra, la investigación se ha llevado a cabo en ambos territorios.

En este sentido, los sujetos seleccionados para las entrevistas en profundidad (caracterizados en todos los casos por sus responsabilidades políticas en el área de educación o por su pertenencia directa a la comunidad escolar) lo han sido en función de su posicionamiento en esas dos líneas de fractura consideradas significativas en la sociedad vasco-navarra. Se han realizado un total de ocho entrevistas en profundidad. Atendiendo al criterio propuesto, se ha hablado, por un lado, con representantes de las siguientes organizaciones políticas: EAJ-PNV (eje derecha-vasca), ARALAR (eje izquierda-vasca) ${ }^{4}$, PSEEE (eje izquierda-española), PP (eje derecha-española) y UPN (eje derechaespañola) $)^{5}$. Por otro lado, también se han realizado entrevistas en profundidad a representantes de los sindicatos que detentan la mayoría sindical, tanto en el ámbito de la educación como en el ámbito sociolaboral general de los dos territorios mencionados: ELA, LAB y STEE-EILAS ${ }^{6}$. Todos ellos se sitúan en la órbita del universo nacionalista vasco, puesto que reclaman un ámbito vasco de relaciones laborales y defienden la utilización vehicular de la lengua vasca en la educación, aunque de manera más atenuada en el caso del sindicato STEE-EILAS, que ofrece una mayor pluralidad interna.

Para el diseño de las entrevistas, se ha utilizado un modelo estandarizado abierto, es decir, se han formulado una serie ordenada de preguntas a todas las

4. EAJ-PNV, Partido Nacionalista Vasco, fuerza política nacionalista vasca de corte demócratacristiano que gobierna actualmente en minoría en la Comunidad Autónoma Vasca. ARALAR, organización política de la izquierda nacionalista vasca, actualmente en la coalición EH Bildu.

5. PSE-EE, Partido Socialista de Euskadi-Euskadiko Ezkerra, organización del PSOE en el País Vasco. PP del País Vasco, organización política del Partido Popular en el País Vasco. UPN, Unión del Pueblo Navarro, organización política conservadora de Navarra que ha mantenido pactos puntuales de colaboración con el PP y que gobierna actualmente en minoría en la Comunidad Foral de Navarra.

6. ELA, Euskal Langileen Alkartasuna, sindicato mayoritario en la CAV y en Navarra en cuanto al número de afiliados, creado hace más de un siglo. LAB, Langile Abertzaleen Batzordeak, sindicato de clase afín al nacionalismo vasco de izquierda. STEE-EILAS, Sindicato de Trabajadores de la Enseñanza de Euskadi, sindicato sectorial de la educación en la CAPV y Navarra. 
personas entrevistadas, y las respuestas han sido abiertas y libres. Las cuestiones propuestas han girado en torno a los siguientes bloques temáticos: educación y lengua; educación e identidad nacional; educación y ciudadanía, y, por último, educación, inmigración y multiculturalismo.

Por otro lado, los discursos extraídos de las entrevistas en profundidad se han completado con la realización de dos grupos de discusión compuestos por docentes de los distintos niveles de educación obligatoria. Dadas las particularidades del tema investigado y las características propias de los sistemas educativos establecidos en la Comunidad Autónoma Vasca y en la Comunidad Foral de Navarra, además de las dos pautas de selección señaladas más arriba (posicionamiento político e identidad nacional), se han tomado en consideración otros criterios complementarios para la composición de los grupos de discusión, a fin de preservar su diversidad: sexo, tipo de centro (público o concertado), nivel educativo (primaria o ESO), materia impartida (con la inclusión necesaria de docentes que han impartido la asignatura de educación para la ciudadanía), cargo académico (dirección, jefatura de estudios y tutoría) y modelo lingüístico (A, B o D).

A este respecto, debe recordarse que, en las redes educativas, tanto públicas como concertadas o privadas de la Comunidad Autónoma Vasca, existen tres modelos lingüísticos legalmente establecidos (A, B y D), en función del porcentaje de español o de euskera utilizado. En el curso 2011-2012, en la Comunidad Autónoma Vasca, el 65\% del alumnado de educación no universitaria estaba integrado en el modelo $\mathrm{D}$, que tiene a la lengua vasca como lengua vehicular. Esta división por modelos existe también en la Comunidad Foral de Navarra, pero sólo en parte de su territorio, ya que se han establecido tres zonas lingüísticas, y en la zona denominada «no vascófona» se desarrolla el modelo $\mathrm{G}$, íntegramente en español. Este modelo era el hegemónico en el citado curso 2011-2012, puesto que aglutinaba al 55,25\% del alumnado, mientras que el $25,65 \%$ del alumnado estaba matriculado en el modelo D, en lengua vasca.

\section{La educación de la ciudadanía según los agentes políticos, sindicales y educativos vascos}

Todos los discursos coinciden en señalar que la cualidad fundamental que debe desarrollar la escuela en su alumnado es aprender a pensar críticamente. Además, y de manera remarcable, la idea de que la educación debe crear ciudadanos libres, autónomos, críticos, solidarios y participativos está bastante generalizada, aunque algunos enfaticen más unos aspectos que otros. En efecto, existe un gran acuerdo en lo referente a los contenidos liberales de carácter general que se deben fomentar. Sin embargo, a pesar de todo lo anterior, la asignatura EDCH no ha despertado el mismo grado de unanimidad.

Desde un punto de vista profesional, hay quien piensa que sus objetivos deberían ser tratados de forma transversal en el currículo y quien considera que, sin esa asignatura, algunos temas no se tratarían de ninguna manera. Pero más allá del papel secundario que, según los enseñantes, desempeña la asignatura 
en el currículo (es una asignatura que "te toca» dar, es una especie de "maría», no se suspende nunca, los padres desconocen qué finalidad tiene, etc.), la línea de separación que diferencia a los discursos políticos sobre la asignatura viene básicamente determinada por el posicionamiento ideológico izquierda-derecha.

Entre las organizaciones políticas y sindicales de estrategia española, el representante del PP afirma que «esa asignatura adoctrinaba a los jóvenes», dando por sentada ya su desaparición, mientras que el discurso más progresista no cuestiona ni el estatus de la asignatura ni sus contenidos y objetivos.

Entre las organizaciones políticas y sindicales de estrategia vasca, también es el partido más conservador, el PNV, quien más pone en duda la eficacia de la asignatura. Por el contrario, las restantes organizaciones la consideran necesaria, y no son, por tanto, partidarias de que desaparezca. Se muestran recelosas, no obstante, de su posible utilización en beneficio de una visión política que vaya en contra de los intereses del nacionalismo vasco:

Estos [en referencia al nacionalismo español] han tenido una crisis de identidad y han puesto en marcha esta asignatura, para, de alguna manera, extender a todos los rincones el modelo español de ciudadanía, para poderlo trabajar de un modo uniforme. Pero esto responde a una razón de estado, simplemente [...] Estoy seguro de que un profesor abertzale [nacionalista vasco] no trabaja los contenidos que aparecen en algunos libros. (LAB)

Por otra parte, la forma de entender la escala de aplicación de la ciudadanía constituye también un elemento de controversia que fracciona los discursos. A diferencia de lo que ocurre con el tema anterior, la línea de fractura de los diferentes discursos viene marcada, en este apartado, por el marco nacional de referencia de los distintos agentes políticos. Así, en el discurso del nacionalismo español, se ensalza la visión cosmopolita, en la medida en que la apelación a una "ciudadanía del mundo" virtual no pone en riesgo la facticidad de la comunidad política estatal:

[...] queremos que sean ciudadanos libres, gente con capacidad de crítica [...] El mundo es muchísimo más grande y, obviamente, yo apuesto por ciudadanos cosmopolitas, por ciudadanos del mundo. (UPN)

En el discurso nacionalista vasco, sin embargo, se desconfía de la existencia de una ciudadanía cosmopolita y se subraya que el acceso a la ciudadanía universal solo puede realizarse una vez confirmada la propia identidad y la comunidad política de referencia, que carece de reconocimiento en el caso de la nación vasca.

No existe un ciudadano cosmopolita o universal [...] solo puedes ser universal si eres vasco. Puedes tener una identidad compleja, plural, con unos elementos más importantes que otros, pero siempre tienes que partir de algún sitio. Es fundamental ser parte de un colectivo [...] eso es la ciudadanía. De ahí te abres al mundo y el mundo enriquece tu ciudadanía particular. (ELA) 
Todo el mundo habla de universalidad [...] ¿Cuál es nuestro problema? Que no tenemos afianzada nuestra identidad, nuestra personalidad ni nuestro futuro [...] Entonces, nos encontramos con el dilema de siempre: lo autóctono y lo universal, lo de aquí y la globalización. (STEE-EILAS)

\subsection{La educación y la identidad nacional en el País Vasco}

¿Debe ser uno de los objetivos de la educación cívica fortalecer la ciudadanía vasca y/o española? La respuesta a esta pregunta se puede interpretar afirmativamente en todos los discursos políticos. Aun cuando parten de distintas premisas en función del posicionamiento nacional e ideológico, estos discursos presentan un punto de coincidencia en la constatación de la intervención de los sistemas educativos en la construcción y el mantenimiento de las identidades nacionales. En el nacionalismo vasco, se considera que el sistema educativo, en tanto que institución del Estado, nunca constituye una entidad neutra (LAB, ELA, Aralar). En el eje nacional español, se reconoce como referente de la escuela una única realidad nacional, precisamente aquella que emana del Estado (PP, UPN). Las posturas del PNV y del PSE-EE, aunque más ambiguas, se suman a esta percepción que vinculan educación e identidad nacional.

El único discurso que se diferencia notablemente de los agentes políticos es el de los docentes (y del sindicato STEE-EILAS, que representa el sentir de muchos de ellos en tanto que sindicato sectorial). Reconociendo la pluralidad de identidades nacionales existentes en la sociedad vasca, muchos profesionales, abocados a encontrarse en el aula con una realidad plural, procuran soslayar el conflicto latente y hacen esfuerzos por evitar «el adoctrinamiento del alumnado». Uno de los recursos utilizados consiste en despojar a las asignaturas potencialmente conflictivas como la EC de toda connotación nacional (pero no eluden la connotación política, porque la lealtad a los valores democráticos, por ejemplo, constituye una idea recurrente en las intervenciones).

Español, vasco, [...] eso no nos concierne; es como la religión, fuera de la escuela. (Grupo 1)

Pasemos ahora a un estudio más pormenorizado, a partir de dos niveles discursivos. Por una parte, estaría el nivel nacional o colectivo. Las organizaciones nacionalistas vascas se esfuerzan en remarcar el conflicto político en torno al derecho del pueblo vasco a formar un estado, algo que el discurso nacionalista español niega porque entiende el País Vasco como parte indivisible de España. En palabras de ELA, esta lucha se extiende a la escuela. Para LAB, hay una lucha de identidades en la escuela y esto se evidencia en las tensiones sobre los contenidos del currículo, el mapa o el nombre de la CAV en vez de Euskal Herria. Y acaba diciendo que el final lo decidirá la correlación de fuerzas. Aralar reconoce esta lucha y reivindica la necesidad de un sistema educativo propio: 
Euskal Herria es una nación. Y como tal nación necesita su sistema educativo. Necesita saber qué quiere enseñar, cuáles son los valores que quiere transmitir. (Aralar)

Más allá de estas visiones generales sobre el reconocimiento de la dimensión nacional de la educación, el análisis pormenorizado de los discursos políticos muestra una radiografía más matizada en la que destacan las diferencias. De un lado, las organizaciones nacionalistas vascas subrayan la perspectiva conflictual del País Vasco como nación sin estado y la necesidad de un sistema educativo propio que transmita sus valores, en tanto que el discurso nacionalista español entiende que el sistema escolar se enmarca en un territorio, el País Vasco, que es una parte indivisible de España. En opinión de los sindicatos nacionalistas vascos (ELA, LAB), esta confrontación simbólica (implícitamente asumida) se ha extendido a la escuela vasca. En consecuencia, en el seno de esta, se produce una lucha de identidades que posee múltiples manifestaciones: tensiones en torno al contenido del currículo, el mapa, el nombre de la CAV en lugar del Euskal Herria, etc. De otro lado, los representantes del nacionalismo español cuentan con la inexorabilidad del Estado y remarcan que el currículo lo establecen el Gobierno y las leyes españolas.

No obstante, junto a la reafirmación de esta diversidad de posiciones de partida, sabedores del carácter sensible y controvertido que tiene el tema en la sociedad vasca, los agentes políticos y sociales de distinto signo buscan en sus discursos la conciliación y el consenso al enfrentar la realidad de la pluralidad de identidades nacionales, conciliación que es desmentida por el propio contexto discursivo. Ocurre, sobre todo, cuando abordan la forma en que la escuela debería acometer la gestión de la diversidad nacional. Así, el PNV reconoce que la escuela debería compaginar y transmitir las dos identidades nacionales, española y vasca (a pesar de que, en su discurso, solo utiliza referencias a la identidad vasca), en tanto que Aralar destaca la necesidad de crear una nueva cultura entre todas las partes (aunque sería un punto de partida para construir la identidad nacional vasca), el PSE-EE reconoce la diversidad nacional (si bien atribuye a la identidad nacional vasca un sentido no deseable de identidad excluyente) y UPN soslaya el problema aduciendo que la escuela no entra en ese debate.

\subsection{Escuela vasca y sociedad multicultural}

Los discursos producidos tanto por los agentes sociopolíticos como por los docentes en torno a la diversidad cultural poseen una orientación inequívocamente liberal. Todos ellos destacan positivamente la pluralidad cultural creada por los recientes flujos migratorios y señalan el enriquecimiento que han generado, entendido este como el beneficio mutuo que se deriva del contacto entre diferentes culturas. La perspectiva liberal aflora igualmente a la hora de reconocer el derecho que tiene la población inmigrada a salvaguardar su cultura de origen y su identidad cultural. En la misma línea, los grupos de profesorado 
no perciben ninguna incompatibilidad entre este derecho y la necesidad de integrar al alumnado inmigrante.

Nosotros, cuando hablamos de diversidad cultural, siempre vamos a hablar en positivo. [...] El tener la oportunidad de que estos chavales puedan tratar y puedan convivir, y puedan estar con niños de Sudamérica, o de Rumanía, o de otros países, o africanos, esa posibilidad de enriquecerse, de que les cuenten cosas de su familia, de su país de origen..., se van a enriquecer los niños inmigrantes pero también se van a enriquecer los niños navarros [...]. (UPN)

Es enriquecedor [...]. En vez de verlo como un riesgo, debemos verlo como un desafío que haya alumnos de otras culturas. (LAB)

¿Cuáles son los límites de la visión liberal sobre la diversidad cultural? El discurso nacionalista vasco establece los límites en dos dimensiones claramente identificables. La primera de ellas está relacionada con la posición hegemónica que, en su opinión, deben mantener la lengua y la cultura vascas en el sistema educativo, puesto que la sociedad vasca se encuentra inmersa en un proceso de recuperación cultural y lingüística. La segunda dimensión que este discurso erige como límite a la diversidad cultural es el componente religioso de dicha pluralidad. En este sentido, se defiende la laicidad del ámbito escolar.

Es enriquecedora la existencia de diferentes culturas, siempre que se respete y se desarrolle la cultura de cada uno. Las culturas se enriquecen relacionándose. De ese modo la multiculturalidad es enriquecedora. Pero, sin forzar ni anular al otro, sin olvidar o diluir las principales bases de nuestra cultura. Que sea siempre un modo de desarrollarla. (ELA)

Los temas y creencias de la fe islámica, es decir, esos espacios que están cercanos al adoctrinamiento no deben tener ningún lugar en el centro escolar. Está bien conocer la influencia de la religión cristiana o la católica en la historia. Es difícil conocer la historia de Occidente sin conocer la influencia de la religión, pero eso no debe ser analizado desde la creencia o la fe. Sin ninguna duda, la escuela tiene que ser un espacio laico, con mayúsculas, y no debe haber ninguna característica religiosa en el centro. Esa función corresponde a la familia, al ámbito privado. (STEE-EILAS)

Los límites de la visión liberal de la diversidad cultural son otros para los discursos situados en el eje español. En ellos, no se problematiza la diversidad lingüística —oficialmente reconocida - del sistema educativo vasco y la enseñanza religiosa en la escuela. Respecto de la primera, este discurso subraya la idea de diversidad «institucionalizada» que se materializa en la institución escolar vasca, fundamentalmente, en la oferta de diversos modelos lingüísticos, tanto en lengua vasca como española. Respecto de la segunda, también valora positivamente el cumplimiento de la ley en relación con la enseñanza de la religión en la escuela y con la contratación de profesores de religión. 
No obstante, en el discurso nacionalista español, también existen fronteras que la diversidad no puede traspasar, aunque estas no se sitúan en el ámbito moral y religioso, sino en el moral y político. Así, los márgenes asumibles de la diversidad se encuentran, en este caso, en la dimensión más política de la educación, pues se considera que dicha diversidad debe venir siempre acompañada por la aceptación de valores de la sociedad receptora considerados esenciales, entre otros, de valores democráticos. El objetivo final es que los inmigrantes sean ciudadanos, por encima o a pesar de las diferencias e identidades culturales. Se observa, de este modo, la importancia que adquiere la idea de la escuela como promotora de lealtad a determinados principios políticos comunes en el discurso del nacionalismo español, por contraposición a la preeminencia que se otorga a la escuela como garante de la cultura vasca común en el discurso nacionalista vasco.

Un ciudadano lo es de una sociedad porque vive en ella, trabaja, se desarrolla, hace cultura, se asocia, paga impuestos... Ese es un ciudadano pleno, me da igual que sea vasco, español, francés... Tiene todos los derechos y todas las obligaciones de esa sociedad compartidas [...] Todos aquellos que vengan de culturas distintas [...], los que vengan de organizaciones sociales diferentes, supuestos distintos, yo creo que también deben aprender en la escuela y en la sociedad el núcleo de nuestros valores, de los valores democráticos. (PSE-EE)

Pero, además de los límites diferenciados que cada uno de los discursos impone a la visión liberal de la diversidad cultural, existe un ámbito que todos ellos (además de los docentes) consideran inviolable a las influencias de esa diversidad de reciente creación: el currículo formal de la institución escolar. Así como prevalece con carácter general el principio liberal de respeto de las culturas foráneas y la libertad para el mantenimiento de estas, no se deriva de esa aceptación inicial una implicación de los poderes públicos y, por tanto, de la escuela en su salvaguarda. En consecuencia, tampoco se ve necesaria la adaptación del currículo escolar, aunque sí se contempla la inclusión informal de determinadas manifestaciones culturales que reflejen la diversidad existente en la sociedad dentro del ámbito escolar (fiestas, celebraciones, relatos de cuentos, etc.).

Yo no sé si hay que proteger esa cultura [de origen]. Yo creo que ni la sociedad ni la escuela tienen la responsabilidad de mantener ni proteger la cultura a nadie. (PSE-EE)

No sé si [las expresiones culturales de los alumnos inmigrantes] tienen que tener un lugar en el curriculo. (ELA)

Yo creo que la mezcla enriquece [...] Las diferencias existen, son intensas porque vienen de otra cultura, y por lo tanto creo que es bueno conocerlas, porque cuando conoces, entiendes, y cuando entiendes, respetas y no desprecias. Esto 
es fundamental, y que en el clima del centro [escolar] se hagan actividades de ese tipo me parece muy bien. Más complicado me parece entrar dentro del curriculo. (PSE-EE)

\section{Conclusiones}

En este estudio, se han tratado de identificar los discursos que determinados agentes cualificados (políticos, sindicales y docentes) construyen en torno a la ECDH y la transmisión de la ciudadanía en los sistemas educativos vasco y navarro. Más allá de las ya conocidas discordancias que suelen manifestarse entre los discursos declarados y las prácticas sociales y educativas que se materializan en la vida cotidiana, se ha considerado que tales discursos poseen interés, en primer lugar, porque recogen de manera suficientemente fiable el modo de pensar de los agentes citados y, por ende, reflejan las distintas concepciones (potencialmente conflictivas) que coexisten en la sociedad vasca; en segundo lugar, porque el hecho de hacerlos explícitos puede permitir poner en cuestión determinadas representaciones estereotipadas sobre el ideario de los agentes implicados, que, a menudo, han impregnado el conocimiento de sentido común. Los discursos analizados se han producido en torno a tres dimensiones consideradas relevantes en la investigación y que aluden a los objetivos morales de la educación cívica, a la escala territorial en la que se comprende (estatal, nacional, cosmopolita, etc.) y a su relación con la diversidad cultural en la sociedad vasca actual.

Los discursos han sido heterogéneos, tanto en lo referente a la situación concreta de la EDCH en el currículo escolar como, en un sentido más general, en lo referente a los contenidos y objetivos relacionados con la transmisión de la ciudadanía en la sociedad vasco-navarra. No obstante, en el análisis, resulta relevante la existencia de una dimensión nuclear compartida por todos ellos. Esta dimensión alude al componente moral de la educación cívica. En torno a ella, se vislumbra una percepción común, de base eminentemente liberal, aunque no excesivamente elaborada, en la que se manifiestan de acuerdo todos los agentes políticos y educativos. En efecto, todos ellos sostienen que el objetivo de la educación cívica debe ser la formación de personas críticas, autónomas, participativas, solidarias y tolerantes. En la misma medida, existe una gran conformidad sobre la dimensión de la cultura, especialmente en la valoración de la (nueva) diversidad cultural, que es considerada enriquecedora y positiva. Las recientes diferencias culturales derivadas de las últimas oleadas migratorias, instaladas ya en la sociedad vasca, constituyen un hecho y un derecho (del ámbito privado, según la mayoría) para todos ellos.

De esta percepción común, surgen discursos más diferenciados, en función de la posición de los distintos agentes. La primera distinción reseñable es aquella que diferencia a docentes y políticos. El discurso del profesorado, que está condicionado por los problemas del ejercicio docente cotidiano, refleja, más allá de la declaración de principios, las dificultades prácticas que conlleva la gestión exitosa de la pluralidad cultural en la escuela (entre otros, la escasez 
de medios materiales e intelectuales), así como la tensión permanente con los valores democráticos que esta cuestión genera en el ámbito educativo. Por su proximidad a la diversidad cultural en el ámbito académico, el discurso de los profesionales es, en general, el menos reticente a ofrecer en la escuela espacios de expresión a las culturas minoritarias de la inmigración más reciente. En contraposición, el discurso de los agentes políticos y sindicales (sin distinción de referente nacional o de posicionamiento ideológico) es optimista en cuanto a la valoración de la diferencia cultural. Sin embargo, el discurso político manifiesta unánimemente su oposición a otorgar a las culturas migrantes cualquier posibilidad de influencia en el ámbito escolar formal, fundamentalmente, en la modificación de los contenidos curriculares. Esta desconfianza es mayor, si cabe, en las organizaciones de la órbita nacionalista vasca (y en algunos profesionales del mismo universo político), que temen el retroceso de la lengua autóctona (socialmente minoritaria y aún no suficientemente consolidada) en la educación.

Por lo que se refiere a los agentes políticos y sindicales, la línea de fractura de los discursos la establece el referente nacional (eje vasco-español) en mayor medida que el posicionamiento ideológico (eje derecha-izquierda). En términos generales, se ha podido identificar un primer discurso que atribuye una dimensión política a la educación cívica, en relación estricta con la preservación de los principios democráticos, y un segundo discurso que otorga preeminencia a la dimensión nacional en la educación de la ciudadanía. Estos discursos corresponden a los agentes políticos del nacionalismo español y a los agentes del nacionalismo vasco, respectivamente. De un lado, los agentes de la órbita española no se ven impelidos a explicitar ninguna lealtad política, puesto que el marco político y legal estatal en el que se inserta el funcionamiento de la escuela garantiza suficientemente dicha lealtad de una forma rutinaria. Es por ello que también se muestran más proclives a asignar objetivos cosmopolitas a la educación. De otro lado, los agentes de la órbita vasca entienden la ciudadanía como un vínculo con la propia comunidad política, en este caso, del País Vasco. Sin embargo, este vínculo carece del sustento de la legalidad y tiene, en consecuencia, dificultades para reproducirse en la vida cotidiana de la escuela. En efecto, es esta la razón por la que el nacionalismo vasco manifiesta, de una forma más o menos implícita, que existen temas pendientes de resolución antes de abrir el debate sobre el cosmopolitismo y el multiculturalismo.

Una muestra de la medida en que esta cuestión irresuelta en la sociedad vasca crea dilemas en la escuela viene dada por la actitud que muestran los profesionales de la educación en torno al tema. En efecto, el análisis del discurso de los docentes revela que determinados componentes de la ECDH (la dimensión nacional, entre otros), que separan y diferencian los discursos de los agentes políticos sobre la educación cívica, además de crear controversia y desacuerdo potencial en la propia sociedad, son soslayados, en la medida de sus posibilidades, por los profesionales en sus prácticas educativas cotidianas. De hecho, los docentes evitan pronunciarse sobre las cuestiones más controvertidas, o lo hacen de forma difusa y ambigua, $y$, en ciertos casos, simplemente las excluyen, 
situándolas al margen de la jurisdicción escolar. Así pues, si la controversia y el debate han sido inherentes a la ECDH a causa de los temas socialmente sensibles que incluye, independientemente del marco social o político de su aplicación, esta investigación viene a corroborar la idea de que el cometido del sistema educativo en la formación de la ciudadanía deviene aún más complejo en sociedades como la vasca, que carecen de un consenso social manifiesto en cuanto a su propia definición.

\section{Referencias bibliográficas}

Andrews, Rhys y Mrсоск, Andrew (2007). «Citizenship Education in the UK: Divergence within a Multi-National State». Citizenship Teaching and Learning [en línea], 3 (1), 73-88. <http://www.citized.info/ejournal>.

Appiah, K. Anthony (2003). «Liberal Education: The United States Example». En: McDonough, Kevin y Feinberg, Walter (eds.). Citizenship and Education in Liberal-Democratic Societies: Teaching for Cosmopolitan Values and Collective Identities. Nueva York: Oxford University Press.

Asari, Eva-Maria; Halikiopoulou, Daphne y Mock, Steven (2008). «British National Identity and the Dilemmas of Multiculturalism». Nationalism and Etnhic Politics [en línea], 14 (1), 1-28. <http://dx.doi.org/10.1080/13537110701872444>.

BANKs, James A. (2008). «Diversity, Group Identity, and Citizenship Education in a Global Age». Educational Researcher [en línea], 37 (3), 129-139. <http://dx.doi.org/10.3102/0013189X08317501>.

Barber, Benjamin (2004). Democracia fuerte. Granada: Almuzara.

BÁrCENA, Fernando (1999). «La educación de la ciudadanía». En: BÁrCena, Fernando; GiL, Fernando y Jover, Gonzalo (eds.). La escuela de la ciudadanía: Educación, ética y política. Bilbao: Desclée de Brouwer.

Bolívar, Antonio (2004). «Ciudadanía y escuela pública en el contexto de la diversidad cultural». Revista Mexicana de Investigación Educativa, 9 (20), 15-38.

Brighouse, Harry (2003). «Should We Teach Patriotic History?». En: McDonough, Kevin y FeInBerg, Walter (eds.). Citizenship and Education in Liberal-Democratic Societies: Teaching for Cosmopolitan Values and Collective Identities. Nueva York: Oxford University Press.

Callan, Eamonn (2004). «Citizenship and Education». Annual Review of Political Science [en línea], 7, 71-90. $<$ http://dx.doi.org/10.1146/annurev.polisci.7.012003.104924>.

Ceballos, José Angel; Escandell, José J. y Páramo, Antonio (2009). Diecinueve manuales de Educación para la Ciudadanía. Madrid: CEU Ediciones.

Colom Cañellas, Antonio J. y Rincón Verdera, Juan C. (2007). Educación, república y nueva ciudadanía. Valencia: Tirant lo Blanch.

Cortina, Adela (1997). Ciudadanos del mundo: Hacia una teoría de la ciudadanía. Madrid: Alianza.

Doppen, Frans H. (2010). «Citizenship Education and the Dutch National Identity Debate». Education, Citizenship and Social Justice [en línea], 5 (2), 131-143. <http://dx.doi.org/10.1177/1746197910370723>.

Etzioni, Amitai (1999). La nueva regla de oro: Comunidad y moralidad en una sociedad democrática. Barcelona: Paidós. 
FAour, Muhammad y Muasher, Marwan (2011). Education for Citizenship in the Arab World. Key to the Future. Washington D. C.: Carnegie Middle East Center.

Galston, William (1993). "Educación cívica en el estado liberal». En: Rosenblum, Nancy (ed.). El liberalismo y la vida moral. Buenos Aires: Ediciones Nueva Visión.

García Guitián, Elena (2008). «La educación cívica como educación política». En: Águila, Rafael del; Escámez, Sebastián y Tudela, José (eds.). Democracia, tolerancia y educación cívica. Madrid: Servicio de Publicaciones de la Universidad Autónoma de Madrid.

Giddens, Anthony (2000). "Citizenship education in the global era». En: Pearce, Nick y Hallgarten, Joe (eds.). Tomorrow's Citizens: Critical Debates in Citizenship and Education. Londres: Institute for Public Policy Research.

Green, Andy (1990). Education and State Formation. Nueva York: St. Martin Press.

Gutmann, Amy (2001). La educación democrática: Una teoría politica de la educación. Barcelona: Paidós.

- (2008). La identidad en democracia. Madrid: Katz Editores.

IcHilov, Orit (2003). «Teaching Civics in a Divided Society: The Case of Israel». International Studies in Sociology of Education [en línea], 13 (3), 219-241. <http://dx.doi.org/10.1080/09620210300200111>.

Innerarity, Carmen (2007). «El islam y la República: Un conflicto entre dos identidades». Papers, 84, 139-147.

Kerr, David (1999). "Citizenship Education in the Curriculum: An International Review». The School Field, 10 (3/4), 5-32.

- (2012). "Comparative and international perspectives on citizenship education». En: Arthur, James y Cremin, Hilary (eds.). Debates in Citizenship Education. Abingdon: Routledge.

Kisby, Ben (2006). «Social Capital and Citizenship Education at the School». British Politics [en línea], 1 (1), 151-160.

<http://dx.doi.org/10.1057/palgrave.bp.4200006>.

Kymlicka, Will (1996). Ciudadanía multicultural. Barcelona: Paidós.

- (2002). «Dilemas de la educación cívica en las sociedades pluralistas». En: Educación, ética y ciudadanía: Actas del IV Congreso Internacional de Filosofía de la Educación. Madrid: UNED Ediciones.

- (2003). «La educación para la ciudadanía». En: La política vernácula: Nacionalismo, multiculturalismo y ciudadanía. Barcelona: Paidós.

Lickona, Thomas (2000). «Educación del carácter: Cultivar la virtud». En: ReigeLuth, Ch. M. (ed.). Diseño de la instrucción: Teorías y modelos II. Vol. II. Madrid: Aula XXI/Santillana.

LORENZO AlQuÉZAR, Rafael (2010). «Educación para la ciudadanía: Reflexiones incidentales». En: Lorenzo Alquézar, Rafael y Benedicto Rodríguez, Rubén (eds.). Educación cívica: Democracia y cuestiones de género. Barcelona: Icaria.

Macintyre, Alasdair (1993). «¿Es el patriotismo una virtud?». Bitarte, 1, 67-85.

MÁiz, Ramón (2003). "Nacionalismo y multiculturalismo». En: Arteta, Aurelio; García Guitián, Elena y Máiz, Ramón (eds.). Teoría política: poder, moral, democracia. Madrid: Alianza Editorial.

Mateos, Txoli (2009). "Los dilemas de la educación cívica en la escuela vasca multicultural». XVII Congreso de Estudios Vascos. Innovación para el Progreso Social Sostenible. San Sebastián: Eusko Ikaskuntza, 991-1000.

Miller, David (1997). Sobre la nacionalidad: Autodeterminación y pluralismo cultural. Barcelona: Paidós. 
- (2000). "Citizenship: what does it mean and why is so important?». En: Pearce, Nick y Hallgarten, Joe (eds.). Tomorrow's citizens: Critical Debates in Citizenship and Education. Londres: Institute for Public Research.

Molina Puche, Sebastián; Miralles Martínez, Pedro y Ortuño Molina, Jorge (2013). «Concepciones de los futuros maestros de Educación Primaria sobre formación cívica y ciudadana». Educatio Siglo XXI, 31 (1), 105-126.

Naval, Concepción (1995). Educar ciudadanos: La polémica liberal-comunitarista en educación. Pamplona: EUNSA.

- (2003). "Orígenes recientes y temas clave de la educación para la ciudadanía democrática actual». Revista de Educación [en línea], número extraordinario, 169-189. <http://dx.doi.org/10.4438/1988-592X-0034-8082-RE>.

Niens, Ulrike, O’Connor, Una y Smith, Alan (2013). "Citizenship Education in Divided Societies: Teacher's Perspectives in Northern Ireland». Citizenship Studies [en línea], 17 (1), 128-141. <http://dx.doi.org/10.1080/13621025.2012.716214>.

Nussbaum, Martha C. (1999). Los limites del patriotismo: Identidad, pertenencia y "ciudadanía mundial». Barcelona: Paidós.

Pearce, Nick y Hallgarten, Joe (eds.) (2000). Tomorrow's Citizens: Critical Debates in Citizenship and Education. Londres: Institute for Public Policy Research.

Pedazhur, Ami (2001). «The Paradox of Civic Education in Non-Liberal Democraties: The Case of Israel». Journal of Education Policy [en línea], 16 (5), 413-430. <http://dx.doi.org/10.1080/02680930110071020>.

Pedró, Francesc (2003). «¿Dónde están las llaves?: Investigación politológica y cambio pedagógico en la educación cívica». En: Benedicto, José y Morán, M. Luz (eds.). Aprendiendo a ser ciudadanos: Experiencias sociales y construcción de la ciudadanía entre los jóvenes. Madrid: Instituto de la Juventud.

Peña, Javier (2009). "El retorno de la virtud cívica». En: Rubio Carracedo, José; Rosales, José M. y Toscano Méndez, Manuel (eds.). Democracia, ciudadanía y educación. Madrid: Akal.

Ramírez, Francisco O. y Boli, John (1999). «La construcción política de la escolarización de masas: Sus orígenes europeos e institucionalización mundial». En: Fernández Enguita, Mariano (ed.) Sociología de la educación. Barcelona: Ariel.

Rosales, José María (2000). «La educación de la identidad cívica: Sobre las relaciones entre nacionalismo y patriotismo». En: Rubio Carracedo, José; Rosales, José M. y Toscano Méndez, Manuel (eds.). Ciudadanía, nacionalismo y derechos humanos. Madrid: Trotta.

Schnapper, Dominique (2001). La comunidad de los ciudadanos: Acerca de la idea moderna de nación. Madrid: Alianza.

Schulz, Wolfram; Fraillon, Julian; Ainley, John; Losito, Bruno y Kerr, David (2010). Estudio internacional sobre educación civica y ciudadana: Marco de la evaluación. Madrid: Ministerio de Educación.

Schwille, John y Amadeo, Jo-Ann (2002). "The Paradoxical Situation of Civic Education in Schools: Ubiquitous and yet elusive». En: Steiner-Khamsi, Gita; Torney-Purta, Judith y Schwille, John (eds.). New Paradigms and Recurring Paradoxes in Education for Citizenship: An International Comparison. Bingley (U. K.): Emerald Group.

Silveira, Pablo da (2009). «Educación cívica: Tres paradigmas alternativos». En: Rubio Carracedo, José; Rosales, José M. y Toscano Méndez, Manuel. Democracia, ciudadanía y educación. Madrid: Akal. 
Smith, Alan (2003). «Citizenship Education in Northern Ireland: Beyond National Identity?». Cambridge Journal of Education [en línea], 33 (1), 15-31. <http://dx.doi.org/10.1080/0305764032000064631>.

Soriano Ayala, Encarnación (2008). «Formando ciudadanos para ejercer la democracia», En: Soriano Ayala, Encarnación (ed.). Educar para la ciudadanía intercultural y democrática. Madrid: La Muralla.

Swimelar, Safia (2012). «Education in Post-War Bosnia: The Nexus of Societal Security, Identity and Nationalism». Ethnopolitics [en línea], 12 (2), 161-182. <http://dx.doi.org/10.1080/17449057.2012.656839>.

Taberner Guasp, José y Bolívar Botía, Antonio (2002). «Introducción». En: Durkheim, Émile. La educación moral. Barcelona: Trotta.

TAmir, Yael (1993). «United We Stand?: The Educational Implications of the Politics of Difference». Studies in Philosophy and Education [en línea], 12, 57-70. <http://dx.doi.org/10.1007/BF01235473>.

Torney-Purta, Judith (2002). "Patterns in the Civic Knowledge, Engagement and Attitudes of European Adolescents: The IEA Civic Education Study». European Journal of Education [en línea], 37 (2), 129-141. <http://dx.doi.org/10.1111/1467-3435.00098>.

Wadell, Madeline (2013). «Citizenship Education in Egypt». Summer Research (Paper 171) [en línea]. $<$ http://soundideas.pugetsound.edu/summer_research/171)>.

Wiborg, Susanne (2000). «Political and Cultural Nationalism in Education: The Ideas of Rousseau and Herder Concerning National Education». Comparative Education [en línea], 36 (2), 235-243.

Williams, Kevin (1995). «National Sentiment in Civic Education». Journal of Philosophy of Education [en línea], 29 (3), 433-440. <http://dx.doi.org/10.1111/j.1467-9752.1995.tb00371.x>.

Zabalo, Julen; Mateos, Txoli e Iraola, Iker (2013). "Conflicting nationalist traditions and immigration: The Basque case from 1950 to 1980». Nations and Nationalism [en línea], 19 (3), 513-531.

<http://.dx.doi.org/10.1111/nana.12025>. 